\title{
TRIBOELECTRIC CHARGE, ELECTROPHYSICAL PROPERTIES AND ELECTRICAL BENEFICIATION POTENTIAL OF CHEMICALLY TREATED FELDSPAR, QUARTZ AND WOLLASTONITE
}

\author{
H. R. MANOUCHEHRI*, K. HANUMANTHA RAO \\ and K. S. E. FORSSBERG \\ Division of Mineral Processing, Luleå University of Technology, \\ SE-971 87 Luleå, Sweden
}

(Received 11 December 2000; In final form 26 January 2001)

\begin{abstract}
The triboelectric charge attributes of the pure feldspar, quartz and wollastonite mineral samples, after contact with plate and cyclone type tribochargers made up of different materials were investigated. The main electrical properties of the minerals, i.e., electrical conductivity and dielectric constant, were measured and their energetic work functions were estimated. In addition, the behaviour of mineral samples in the electric field of a free-fall separator before and after chemical treatment with different organic reagents was deduced.

The results reveal that the cyclone tribochargers are more effective in imparting charges on mineral grains. The reproducible charges with higher magnitudes are created when cyclones are used for imparting charges on mineral grains. Furthermore, in spite of having relatively similar energetic structures for the three minerals, the Teflon tribocharger demonstrates the feasibility of quartz separation from wollastonite and feldspar. The salicylic acid conditioning promotes feldspar-wollastonite separation.
\end{abstract}

Keywords: Electrical separation; Triboelectric charge; Cyclone tribocharger; Chemical conditioning; Fermi energy; Dielectric constant; Electrical conductivity

*Address for correspondence: Docentvagen 26, SE-977 52, Luleå, Sweden. e-mail: hmanouchehri@yahoo.com 


\section{INTRODUCTION}

Although the basic principles of triboelectric charge and separation of minerals have been defined and the advantages of using such a dry separation method are evident, the commercial applications of the method has not met with success, except for potassium salts and its associated minerals. The main reason is the lack of reproducibility of the charging of the feed minerals due to the fact that tribo-electrification is affected by different factors related to both the surfaces of the minerals and the surrounding ambient conditions [1-6].

During tribo/contact charging and separation the main contributions to determine the particle trajectories are both the sign and magnitude of the charges acquired by mineral grains. However, these two change variously and particles thus behave differently.

Particles traverse paths according to the balance of forces, viz. the electric force, which is the result of interaction between the electric field of the separating device and the electric charges accumulated on mineral grains and gravitational and other co-acting forces. However, the sign and magnitude of the acquisition charge change from one particle to the other and from one test to another, even for the same mineral sample, due to the particle size and shape, contact time, type of contact, surface contamination and impurities, point defects, as well as the surrounding ambient condition. Therefore, the prediction of particle trajectory through the electric field of a separator and as a result the electrical beneficiation potential of a mineral within a mixture becomes more complicated and far from completely understood.

It is generally assumed that during contact/tribo electrification the electrons are responsible for the charge transfer, although ions are mentioned to be responsible in some cases, especially when organic insulators are taken into account [7-9].

It is understood that the direction of the charge transfer is governed by the relative magnitude of work functions of the contacting bodies. So the work function difference of mineral particles and tribocharging medium is the main contribution to determine the sign of the acquisition charge. However, the magnitude of this charge is a complex function of the work function values of the contacting bodies, particle size and shape, time duration and the type of contact, electrical 
properties, e.g., conductivity, of materials brought into intimate contact, as well as the surrounding ambient conditions.

It must be taken into account that the work function of a mineral particle, especially on surface, is affected by the electronic structure of its surface, particle size and shape, lattice defects, impurities, surface contamination and so on $[10,11]$.

\section{Electrical Characteristics of Minerals}

The surface energy structure of semi-conducting minerals, as an extensive class of natural chemical compounds, can be described by the band theory. The position of the Fermi level in the forbidden gap region, electron work function, width of the forbidden gap, the concentration and mobility of the charge carriers, i.e., either electrons or holes, are taken into account by the theory.

Minerals with different energetic levels donate or accept electron(s) when they are brought into intimate contact based on the fact that their work functions are lower or higher than the contacting partner. The charge exchanges continue till the Fermi energies of the contacting bodies become coincident. Finally, when the contact ceases, materials will be left with the same magnitudes but different signs of charges. However, if one or both contacting bodies are good conductors the charge will be lost soon after disconnection as a result of high surface conductance. However, the charges will remain on semi-conducting or insulating materials. The final charge that remains on the surface is defined by the charge back-flow which is a function of material properties, e.g., its conductivity, surrounding ambient conditions, material geometry and roughness, time and speed of surface separation, etc.

The Fermi energy level and the conductivity of a particular semiconducting mineral are defined by the concentration and mobility of the charge carriers of either type of semiconductors as the follows $[10,12]$ :

$$
\begin{aligned}
& \mathrm{E}_{\mathrm{f}}=\left[1 / 2 \mathrm{E}_{\mathrm{g}}-\mathrm{kT} \ln (\mathrm{p} / \mathrm{n})\right] \\
& \sigma=\mathrm{e}\left(\mathrm{p} \mu_{\mathrm{p}}+\mathrm{n} \mu_{\mathrm{n}}\right)=\sigma_{\mathrm{o}} \exp \left(-\mathrm{E}_{\mathrm{g}} / 2 \mathrm{kT}\right)
\end{aligned}
$$

where $\mathrm{E}_{\mathrm{f}}, \mathrm{E}_{\mathrm{g}}, \mathrm{k}, \mathrm{p}, \mathrm{n}, \sigma, \sigma_{\mathrm{o}}, \mu_{\mathrm{p}}, \mu_{\mathrm{n}}$, and e are Fermi level and forbidden gap energies $(\mathrm{eV})$, Boltzman's constant, holes and electron concentrations $\left(\mathrm{m}^{-3}\right)$, conductivities at defined temperature and absolute zero 
$\left(\mathrm{S} \mathrm{m}^{-1}\right)$, hole and electron mobilities $\left(\mathrm{m}^{2} \mathrm{~V}^{-1} \mathrm{sec}^{-1}\right)$, and electronic charge respectively.

The above equations indicate the fact that the electro-physical characteristics of semi-conducting materials, especially on their surfaces, are affected mainly by the concentrations and mobilities of the charge carriers thus, their capability to accept or donate electrons can be modified. However, the modification for specific mineral is limited by the width of its energy gap, i.e., the higher the width of gap energy, the greater is the capability of mineral to be modified. Minerals having narrow forbidden gap within their energy bands are thus barely modified by different pre-treatment methods.

\section{Modification of the Electro-physical Properties of Minerals}

Surface treatment methods, e.g., thermal, chemical, irradiation, doping, and humidity modification, are suggested in order to improve the possibilities for separating different minerals from each others when an electrical separation technique is utilized.

The sign and magnitude of tribo-electric charge acquired by mineral particles under standard conditions can be altered or even artificially modified in a reproducible manner by adsorption of different conditioning agents. The phenomena have been studied in details and observed for some mineral systems [13-16].

An addition of oxidizing or reducing agents changes the equilibrium distribution of electrons through the valance states of ions and electrons of the whole system $[17,18]$. The addition of oxidizing agents on mineral surface decreases the concentration ratio of electrons and holes and the position of Fermi level, while the reducing agents increase these two parameters. Thus the probability of transferring an electron from the valance band of the minerals increases and decreases since the position of the Fermi level changes through the width of the forbidden gap. Adsorption of ionic and cationic reagents by mineral surfaces decreases and increases the thermoionic work function of minerals. So the capability of minerals to be charged negatively or positively will be affected. According to a simple triboelectric theory a mineral must posses a work function greater than charging surface when it is negatively charged and vice versa.

Adsorption of hydrophobic or hydrophilic surfactants on mineral surface gives an opportunity to decrease or increase the avidity of 
mineral surface to adsorb water molecules. Therefore the conductivity and as a result the magnitude of the acquisition of charge can be modified by selective adsorption of these kinds of surfactants. When such an adsorption occurred, the electrical electrophysical characteristic of the minerals should be modified by adjusting the relative humidity of the ambient or even by spraying an ionic solution on mineral surfaces [19]. Such a treatment method is more favourable when conductive-induction and corona type separators are utilized for separating a mixture.

For separating silicates by electrical separation techniques, different investigators made their efforts to find the best conditions for a successful separation. For example the use of HF under different environmental conditions is suggested for separating quartz from feldspar. However, the use of hydrofluoric acid, sulphuric acid, organic acids having hydrocarbon group of sufficient length including salicylic, phtalic, heptylic and cresylic acids, etc., are recommended for separating different silicates, e.g., sillimanite from quartz and biotite, kyanite from quartz-muscovite-chlorite, etc. [1,2,20].

\section{EXPERIMENTAL}

\section{Materials}

Pure crystalline quartz, feldspar (orthoclase) and wollastonite were obtained from Gregory, Bottley and Lloyd. Ltd, UK. These minerals were ground by using jaw and roller crushers respectively, and classified within a different particle size fractions however, the particle size range of $-710 \mu \mathrm{m}$ were considered for separating tests.

The chemical reagents, i.e., sodium oleate, sodium stearate, salicylic acid, benzoic acid and stearyl amine acetate were obtained from different resources and used for conditioning of the minerals.

\section{METHODS}

\section{Charging Minerals and Charge Measurements}

At least two different categories for tribocharging can be assumed in real separation process, i.e., particle-media and particle-particle 
contacts. Therefore two different methods for imparting charge on minerals were considered, i.e., single particle charge where a particle contacts the plate or slides on it, and a cyclone tribocharger in which a mass of particles are present and particles are able to contact the wall of the cyclone and each other. The latter is more familiar with the real separation process, however the contact between the tribocharger and mineral grains seems to be more dominant.

The Faraday well connected to Keithely Electrometer was used for detecting the triboelectric charges acquired by mineral grains.

\section{Electrical Conductivity and Dielectric Constant Measurements}

A Hewlett Packard 4342A Q-meter was used to measure the main electrical properties of mineral samples since both the magnitude and the polarity of the acquisition triboelectric charge are affected by these two parameters to some degree. The Landau and Lifshitz equation [21], suggested for the permittivity of a mixture, was used to correct the measured dielectric constants because of the porosity of the mineral powders.

It is proven that both the conductivity and dielectric constant are frequency-dependent. When the frequency of the measuring device increases the measured dielectric constant decreases. Both the conductivity and dielectric constant increase when the temperature increases $[22,23]$. The measurements were done in room temperature and with the fixed frequency of $100 \mathrm{kHz}$.

\section{The Chemical Conditioning Procedure}

Different Pyrex glass beakers containing dissociated chemicals in the distillated water were obtained. Mineral samples were added to the bekers and the slurries were slowly rotated using mixer. The conditioning time and the amount of chemicals were about 15 minutes and $500 \mathrm{~g}$ per ton respectively for all experiments. After conditioning, the slurries were filtered and dried. However, for preventing undesirable effects of high temperatures a desiccator with temperature of $50-60^{\circ} \mathrm{C}$ of the air circuit was used to dry the mineral samples. 


\section{Triboelectric Separation Tests}

Steel and Teflon micro-cyclones and a aluminium tribocharger (i.e., a bent aluminium tube) were used as the tribochargers for electrical separation tests. Mineral grains were passed through the electric field of a laboratory free-fall electric separator (Fig. 1) after undergoing a contact with tribochargers. On the basis of the mass of differently charged particles accumulated in three bins of the separator the electrical beneficiation potential for each mineral sample was defined.

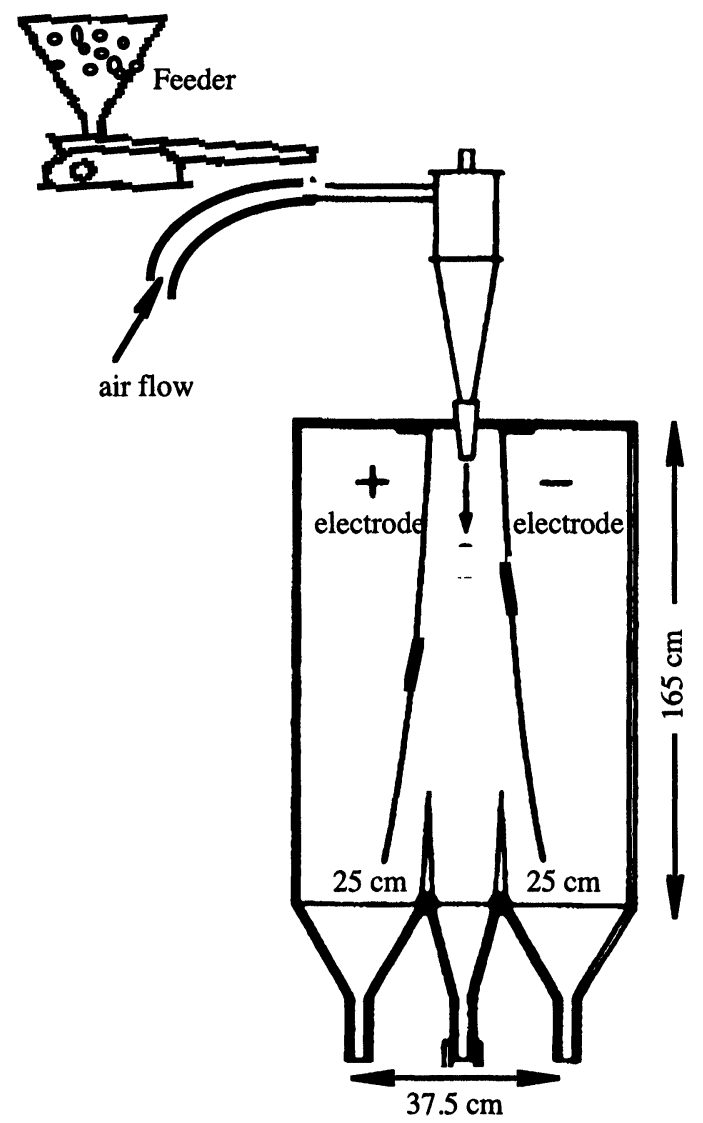

FIGURE 1 The general description of the separating device and the separation procedure. 
According to the first series of tests the feed rate, the cyclone pressure and the electric field of separator were optimized. So the potential of $30 \mathrm{kV}$ and the air pressure of 1.5 bar were used. In addition, in order to avoid of reduce the role of humidity of the surrounding ambient environment both minerals and tribochargers were heated up to $45-50^{\circ} \mathrm{C}$.

\section{RESULTS AND DISCUSSION}

\section{Dielectric Constant and Electrical Conductivity of Mineral Samples}

Figure 2 shows the results of the dielectric constant and electrical conductivity measurements. The values of electrical conductivities for quartz, feldspar and wollastonite are about 58,710 and $580 \mathrm{nS} \mathrm{m}^{-1}$ respectively. However, their dielectric constants are found to be 4.40 , 5.36, and 5.65. These measured values are in a good agreement with some reported data and indicate that the mineral, samples posses similar electro-physical properties [24-26]. This is the why these minerals cannot be separated from each others by conductive induction and corona charge separation techniques. These results
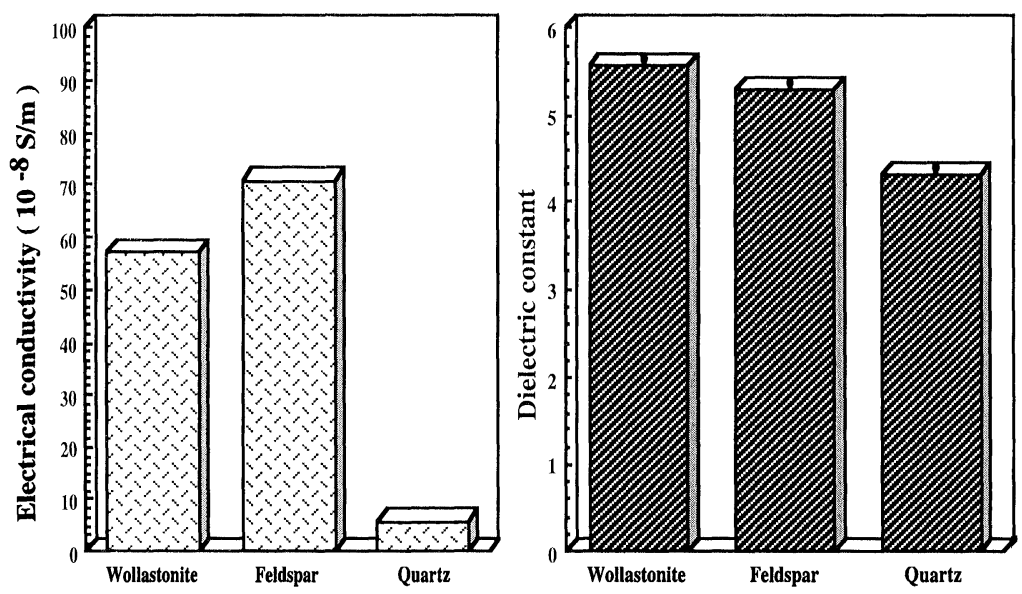

FIGURE 2 Electrical conductivity (left) and dielectric constant (right) of mineral samples. 
indicate that quartz tends to be charged negatively if it contacts two other minerals. This means that the highest magnitude of negative charges should be observed for quartz.

According to the values of the dielectric constant for mineral sampled one can predict that quartz must be charged negatively when it contacts two other minerals. However, wollastonite must hold the positive charge polarity when it contacts quartz and feldspar. This can be explained by the Coehn's rule, which was formulated by Beach [27]. However, the phenomenon is of a limited value due to the difficulties in determining the effective relative permittivity of the surface layer which is often different from the bulk and to the fact that triboelectricfication is based on electronic properties of solids rather than on ionic one.

Dielectric constant for a mineral does not change in a wide extent. However its electrical conductivity may change over about three orders of magnitude which is based not only on the experimental conditions but also on crystallographic features of a mineral the existence of the impurities in crystal lattice and so on [26]. It is also understood that dielectric constant and electrical conductivity of a mineral change as a result of the mineral sample characteristics, such as particle size and shape, irregularities, crystal defects, impurities, porosity of the sample, etc.

\section{Triboelectric Charge Characteristics of the Minerals}

Table I contains the results of the charge measurement of single particles of mineral samples at room and $50^{\circ} \mathrm{C}$ temperatures. Aluminium, copper, nickel, steel and PVC plates were used as the tribocharging in media.

The charge measurements of single particle, indicate that all mineral samples have tendencies to be charged negatively when they contact metals at both room and elevated temperatures, except in the case of wollastonite and feldspar when they contact aluminium plate at room temperature. The latter observation could be the result of the effect of humidity of the surrounding environment. In some cases both polarities of triboelectric charges were monitored for a specific mineral which could be the result of the fact that in some cases the rubbing or the sliding is predominant. So either the charge polarity, charge 
TABLE I The average charge-to-mass ratio for single particle $(1 \mathrm{~mm}<P<2 \mathrm{~mm})$ mineral sample $(\mathrm{nC} / \mathrm{g})$

\begin{tabular}{lllll}
\hline Tribocharger/Temperature & & Feldspar & Quartz & Wollastonite \\
\hline \multirow{4}{*}{$\mathrm{Al}$} & Room temp. & $0.029^{*}$ & -0.026 & $0.01^{*}$ \\
& $50^{\circ} \mathrm{C}$ & -0.835 & -4.26 & $-0.093^{*}$ \\
$\mathrm{Cu}$ & Room temp. & -0.085 & -0.133 & $-0.015^{*}$ \\
& $50^{\circ} \mathrm{C}$ & -1.62 & -1.33 & $-0.164^{*}$ \\
$\mathrm{Ni}$ & Room temp. & -0.174 & -0.465 & $-0.039^{*}$ \\
& $50^{\circ} \mathrm{C}$ & -3.26 & -9.04 & -1.56 \\
$\mathrm{Steel}$ & Room temp. & -0.226 & -1 & -0.067 \\
& $50^{\circ} \mathrm{C}$ & -1.54 & -2.92 & -1.62 \\
$\mathrm{PVC}$ & Room temp. & 5.16 & 7.4 & 7 \\
\hline
\end{tabular}

* Both polarities have been detected for charges.

magnitude or both should be affected. The same has been reported by others [7].

Furthermore the charge increases with increasing temperature. It is result of the reduction of the effects of humidity on the surfaces of both the minerals and tribochargers. The most common mechanism whereby the relative humidity is believed to influence charging is to help increase the surface conductivity of the particles. Therefore the acquired charge will easily drain off to the earth when in contact with the earthed surface. However, it is also proven that the values of the work function as the most important contribution to the governing entity of the contact charging, are reduced by increasing the humidity of both the particles and the tribocharging surfaces [18, 28].

As a result of the high value of the work functin of PVC all mineral grains acquire positive charges when they contact it. However, the charge increases with increasing temperature.

Table II comprises the measured charges for different size fractions of mineral samples when cyclones are utilized for imparting charges. The results indicate that metals induce negative polarity of charges on mineral grains. Furthermore, higher magnitudes of negative charges were observed when minerals contact Perspex due to the fact that Perspex holds the lowest work function value about $2.7 \mathrm{eV}$ [29].

The polarity of charges is positive when Teflon cyclone is the tribocharging medium. However the polarity for quartz is converted to the negative value at elevated temperatures. Tests with the same materials when the minerals were heated up to $100^{\circ} \mathrm{C}$ and $150^{\circ} \mathrm{C}$ 


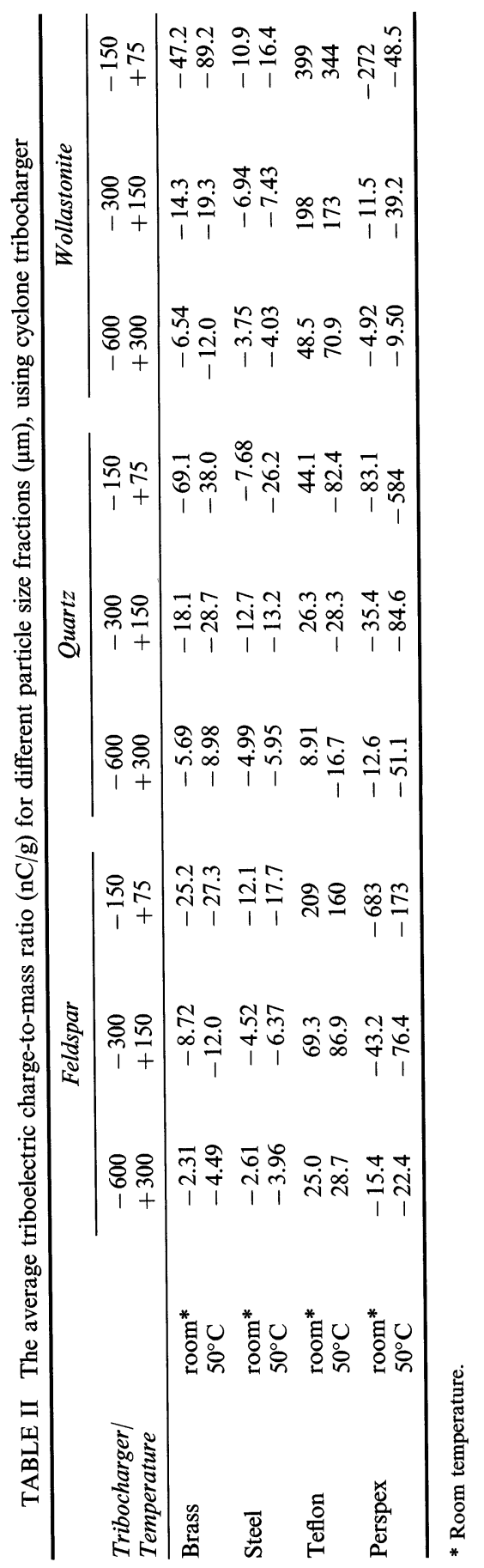


showed the same behaviour for quartz, i.e., its acquired charge was negative, but no changes were monitored for wolastonite and feldspar.

Moreover, the monitoring of triboelectric charges induced by cyclones indicates that the magnitude of these charges is rather higher than single particle charges which should be because of the impact resulted from air pressure as well as the probability of particle/particle contacts. No changes for the polarity of the charges were observed after repeating the tests and the charge magnitude varies within a narrow range so they have contemptible standard deviations. Consequently they are more reliable and reproducible.

Furthermore, the results indicate that feldspar and wollastonite have almost same triboelectric charge characteristics. Quartz however behaves somewhat differently. It seems that these minerals can be ordered in a series based on their work function values. It is thus predicted that quartz and wollastonite posses the highest and the lowest work functions respectively, besides the differences are very low. From the results it can be expected that Teflon tribocharger is the best medium for imparting charge and separating quartz from feldspar and wollastonite. Aluminium tribocharger may be suitable for the same propose or even for separating wollastonite from feldspar. Finally it must be added that the acquisition of charges by minerals is in a good agreement with their electrical properties, especially their conductivities. It suggests that the lower the conductivity, the higher is the detected triboelectric charge.

\section{The Estimation of the Work Function Values for Mineral Samples}

It is widely understood and accepted that the magnitude and polarity of the contact charging are work function-dependent and a linear relationship has been found between the magnitude of triboelectric charges accumulated on surfaces and the values of their work function [30-32]. It could be possible therefore to estimate the value of the work function of one partner by knowing the work function of the other. The trustworthiness of the attained work function is dependent not only upon the experimental precision but also on the accuracy of the known values of the work function.

Accordingly, both the measured charges, i.e., single particle and mass charges imparted by different plates and cyclones respectively, 
were considered and plotted against the values of the work function of tribocharging material chosen from literature.

The magnitude of work functions for metals vary within a wide range and different value of the work function were reported for a metal even by a single researcher. These reported values vary as a result of different experimental methods as well as experimental conditions [33-36]. For metals the values are normally vary between 4 and $5 \mathrm{eV}$. However, the values of the work function for insulators, such as PVC, vary in narrow ranges and are generally greater than the values of metals.

Figure 3 shows the plotted charges acquired by single particles of mineral samples under two different conditions, i.e., room and $50^{\circ} \mathrm{C}$ temperatures, as a function of the value of the work function of the tribocharging media. For all cases the lines with correlation coefficients greater than $82 \%$ are met. The results would be the best if the values of the work function for tribochargers were estimated in situ based on experimental conditions.

The estimated values at the room temperature are 4.394, 4.366 and $4.350 \mathrm{eV}$ for quartz, feldspar and wollastonite, respectively. However, these values increase to $4.512,4.421$ and $4.384 \mathrm{eV}$ for the same mineral
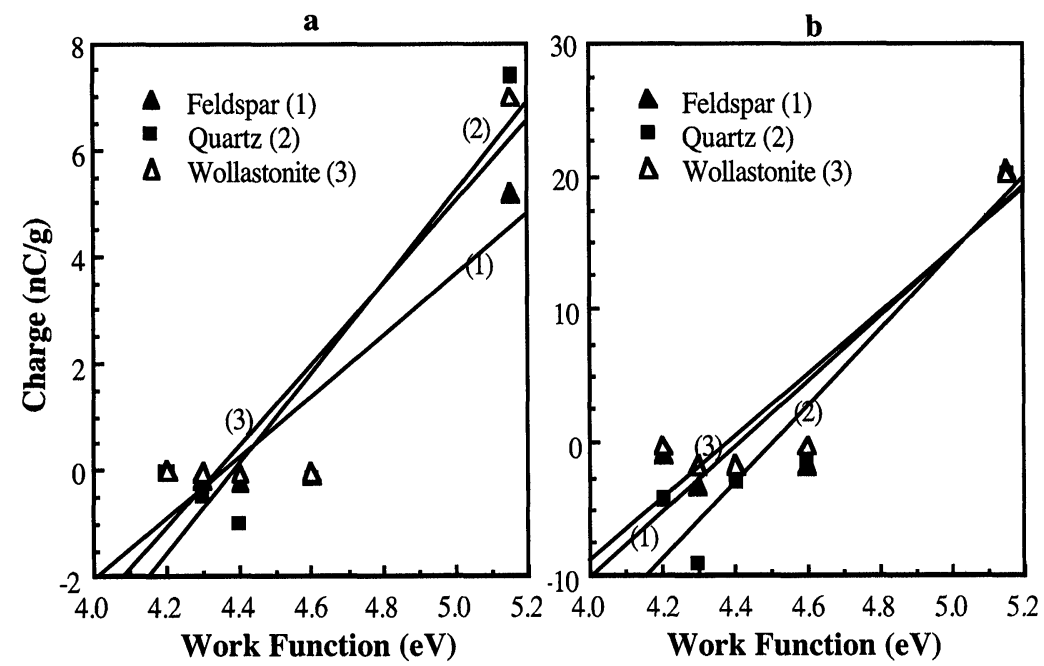

FIGURE 3 Charges acquired by mineral samples as a function of work functions of tribochargers at room temperature $a$ and $50^{\circ} \mathrm{C} b$. 
grains when the temperature is raised to $50^{\circ} \mathrm{C}$ due to the decreasing humidity of the surrounding ambient and the increasing magnitude of the acquired charges. These estimates for the values of the work function of mineral samples were constructed on the basis of deliberated work functions of $4.2 \mathrm{eV}$ for aluminium [34], $4.3 \mathrm{eV}$ for nickel [36], 4.4 for steel [18], 4.6 eV for copper [18,34], and $5.15 \mathrm{eV}$ for PVC [37].

Figure 4 depicts the plotted charges acquired by different size fractions of mineral samples as the work function of cyclone tribochargers at room and $50^{\circ} \mathrm{C}$ temperatures where the work functions of $4.4 \mathrm{eV}$ for steel, $4.2 \mathrm{eV}$ for brass, and $2.7 \mathrm{eV}$ for Perspex were considered. For both the quartz and feldspar mineral samples good correlations were found between the measured charges and work functions of the tribochargers. However, wollastonite behaves differently and generally the correlation coefficients for different size fractions vary in a wide range.

The estimated work functions for quartz vary in the range of 4.399 to 4.815 and 5.476 to 5.508 for $50^{\circ} \mathrm{C}$ and room temperature measurements respectively. However, for the feldspar mineral these ranges vary from 4.521 to 4.68 and from 4.353 to 4.616 individually.

Since the effects of relative humidity of the surrounding ambient can be reduced or even omitted by heating both the minerals and tribochargers, the estimated values when both minerals and tribochargers are heated up to $50^{\circ} \mathrm{C}$ should be more reliable. Accordingly the average estimated values of the work functions for quartz and feldspar are 4.6 and $4.58 \mathrm{eV}$, respectively, These are in a good agreement with the reported values $[4,29,36]$. For wollastonite, as described before, the estimated work function varies widely. However the most reliable value for its work function should be $4.48 \mathrm{eV}$.

The estimated work functions testify that quartz has a higher value of the work function than two other minerals so its avidity to accept electron is higher. Consequently quartz must possess more negative surface charge characteristics with a higher magnitude when it contacts metals. On the other hand wollastonite holds a lower work function and the magnitude of its acquired charges when it contacts different metals must be lower. These observations are in agreement with the results of the charge measurement. The magnitude of the estimated work functions are somewhat higher when the cyclone charging data are used. The main reason would be the particle size 

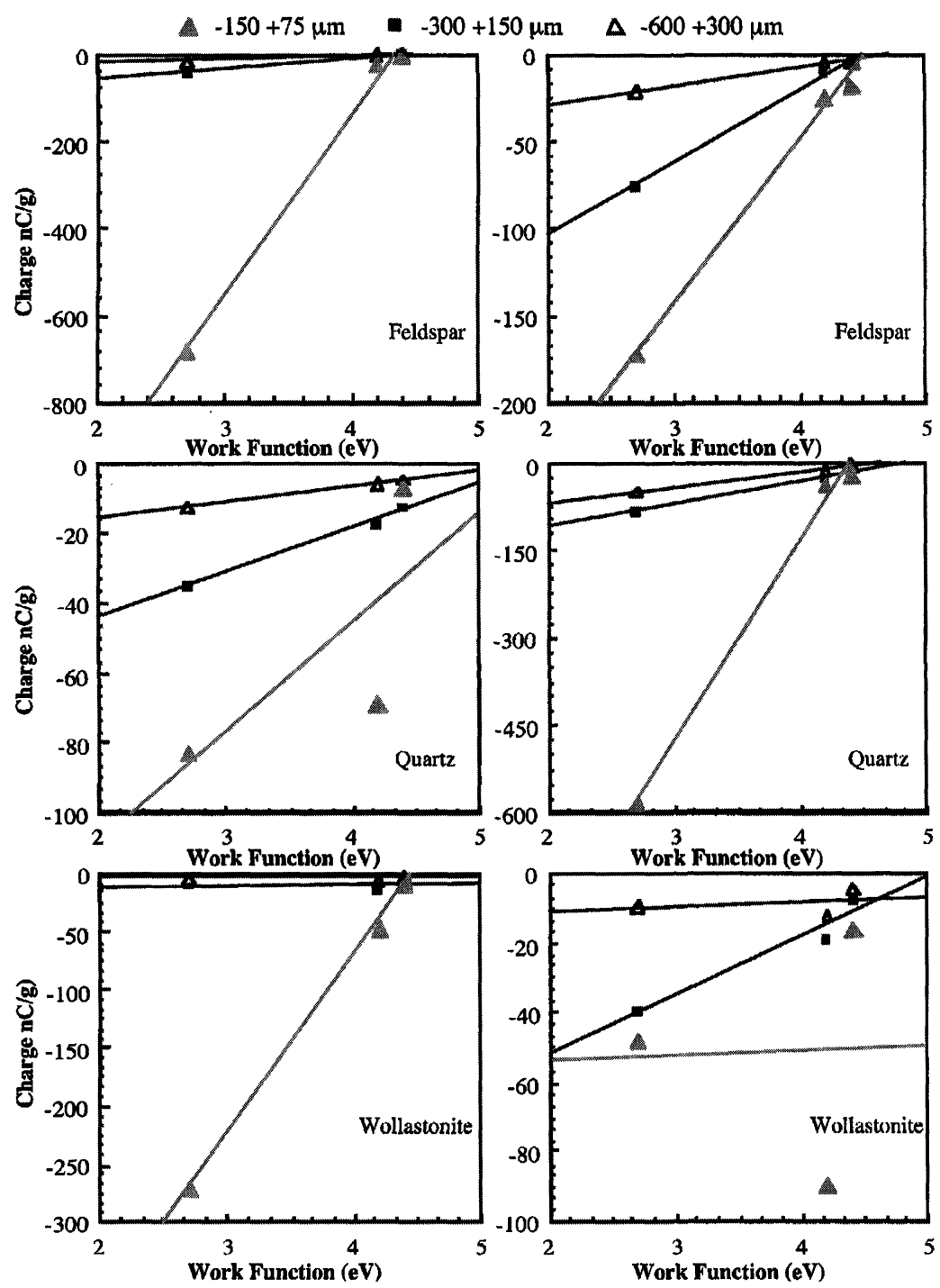

FIGURE 4 The charge acquired by different grains of minerals as a function of the work function of cyclone tribochargers, at room temperature (left) and $50^{\circ} \mathrm{C}$ (right).

dependency of the work function for insulating materials which is illuminated by Gallow and Lama [38]. According to their model the work function increases with decreasing particle size. 


\section{Electrical Separation Results}

Mineral samples after and before conditioning were triboelectrically charged and passed through the electric field of a separating device. The mass of differently charged particles occupying positive, neutral and negative, compartments of the separator were considered as the electrical beneficiation potential for each mineral sample. In order to avoid or reduce the humidity effects and to prevent the undesired influence of temperature on chemicals both minerals and tribochargers were heated up to $50^{\circ} \mathrm{C}$ during the separation process.

According to the results, quartz has more avidity to accept electron from aluminium (Fig. 5). However, feldspar behaves differently which could be because of the humidity effect. Despite the fact that by heating mineral samples and the charging system the effect of humidity is reduced, it cannot be completely omitted. So the feldspar can behave as an electron donor because of humidity

This phenomenon was investigated and reported by Parks et al. [39]. Their experimental results indicated that at the relative humidity about $20 \%$ or even less feldspar acquired positive charge by contacting an aluminium plate. No significant differences were observed by contacting chemically treated minerals with an aluminium tube except for the case of feldspar when salicylic acid was used. In this case feldspar behaved differently and showed accepting properties. In addition, it can be concluded from the separation results that the avidity to accept electrons for all mineral samples increases after using amine. This is because of the fact that amines introduce positive ionic species on mineral surfaces, reducing thus their surface work functions.

Figure 6 shows the separation results of conditioned and non-conditioned mineral samples when they were charged by a steel cyclone. It can be deduced from the this figure that, except for the non-conditioned feldspar, in all other cases the negative charges on mineral grains are predominant. However, the capability of minerals to be charged negatively is greater when amin is used for conditioning.

The responses of minerals to the electric field of a separator when Teflon is utilized for imparting charge are shown in Figure 7. The results are interesting and indicate that without conditioning there is a possibility to separate quartz from wollastonite and feldspar, Both feldspar and wollastonite possess positive charges whereas quartz 

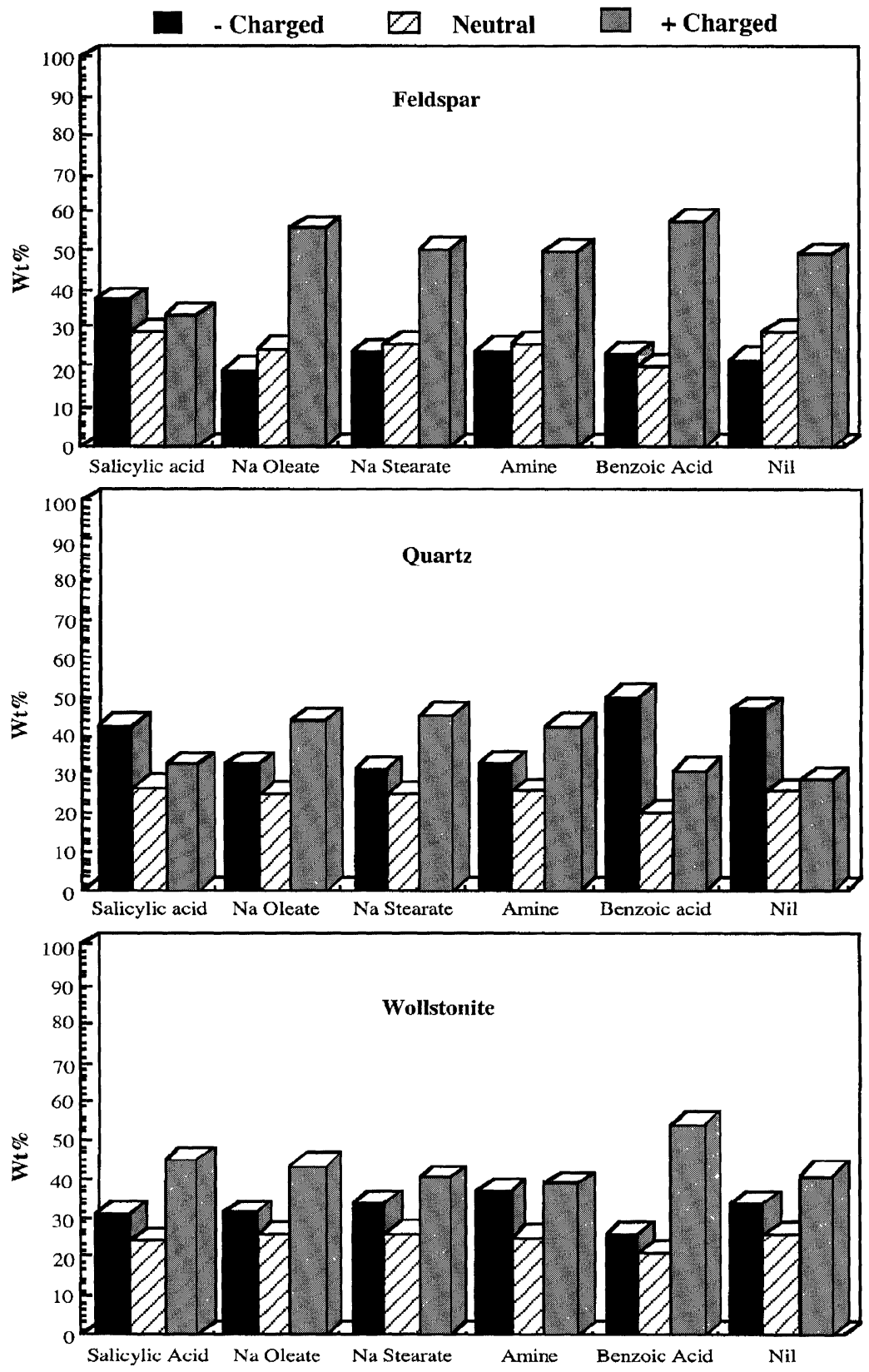

FIGURE 5 The mass of differently charged particles accumulated in different compartments of the separator, using an aluminium tribocharger. 

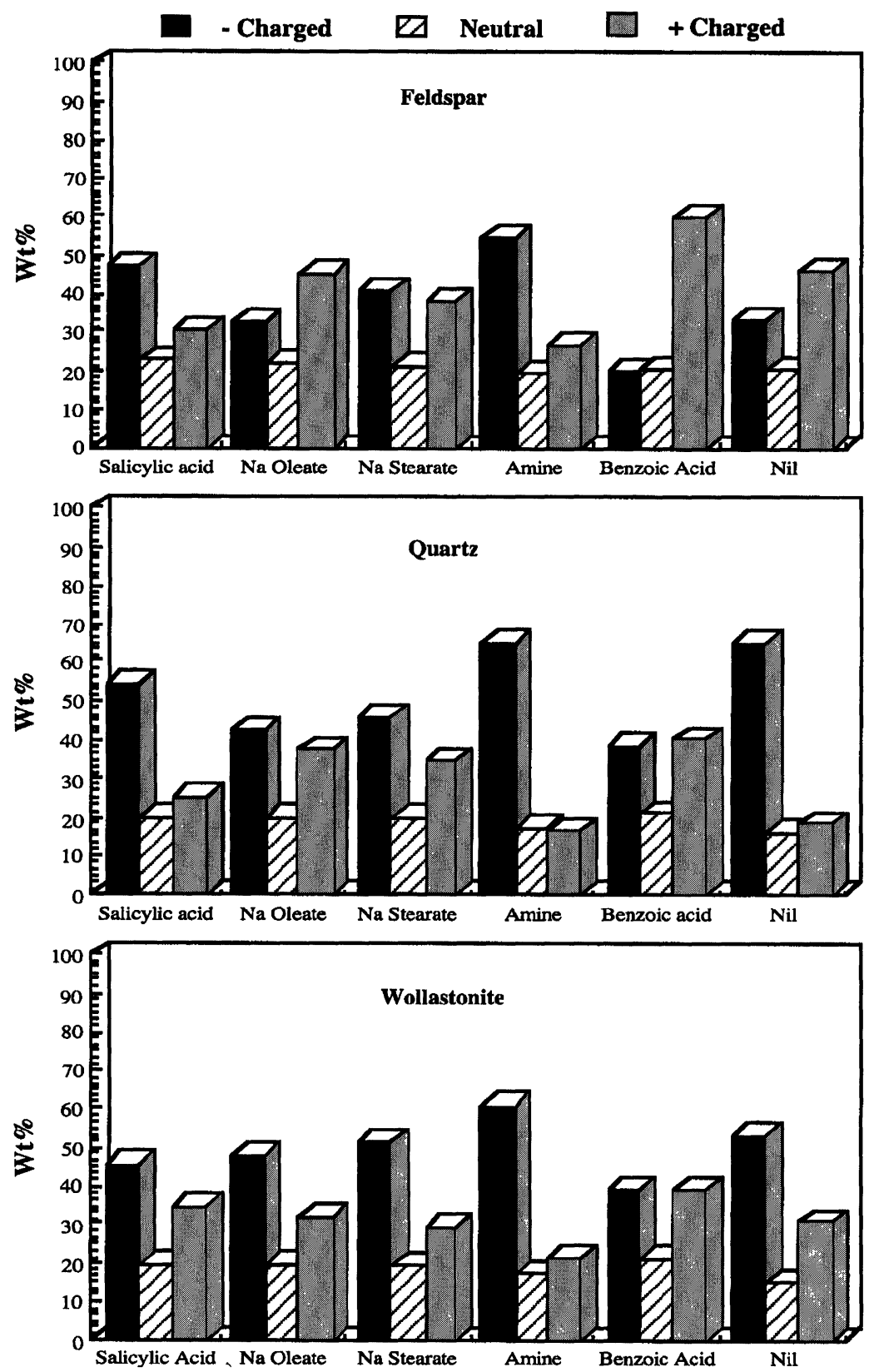

FIGURE 6 The mass of differently charged particles accumulated in different compartments of the separator, using a steel tribocharger. 

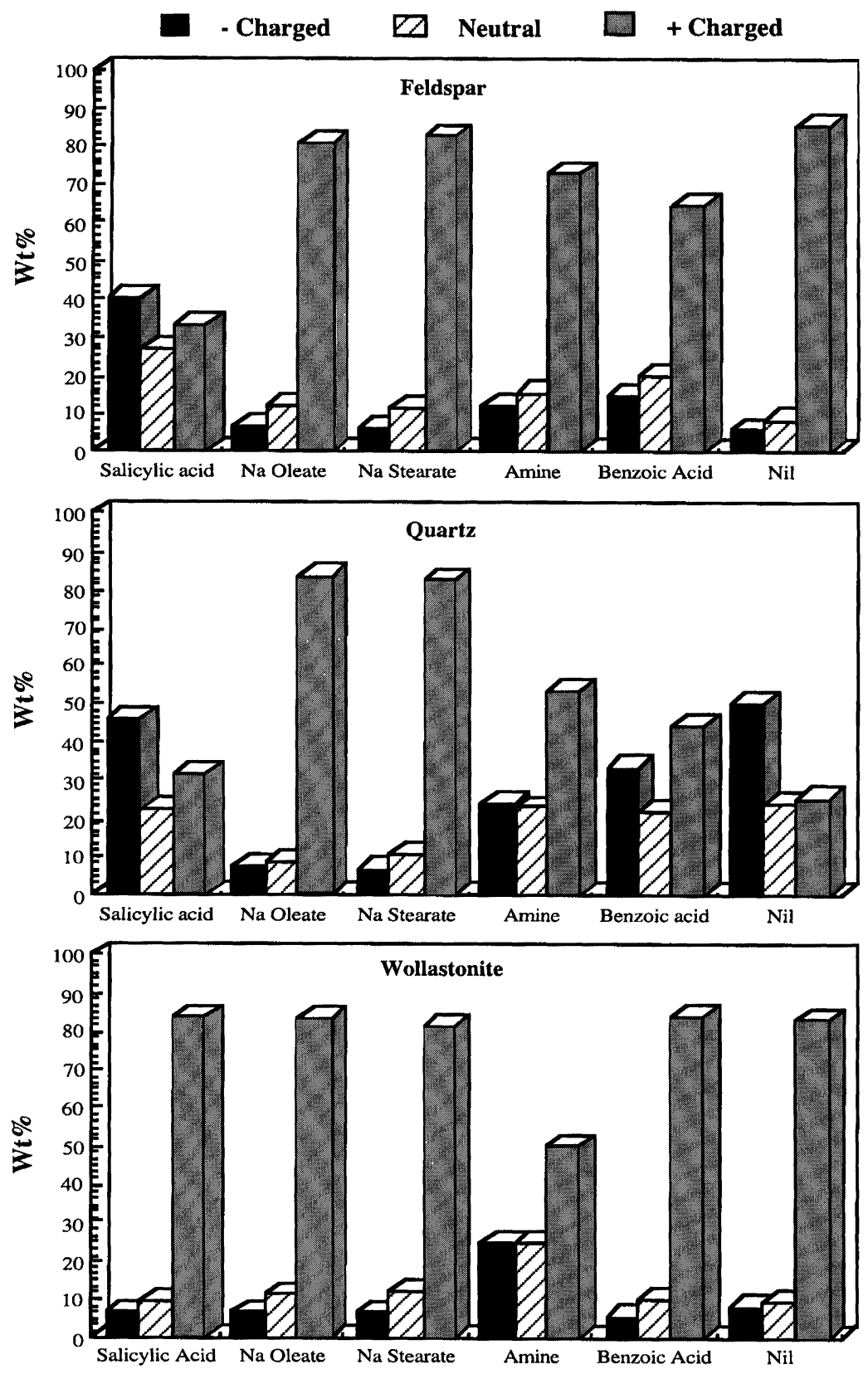

FIGURE 7 The mass of differently charged particles accumulated in different compartments of the separator, using a Teflon tribocharger. 
behaves in an opposite way. In addition, capability of quartz to be charged negatively decreases after chemical conditioning. However the effects of oleate and stearate are significant.

It seems that the value of the work function of Teflon is very close to that of quartz. By adsorption of the negative ionic species of stearate and oleate, the work function of quartz decreases and it gains positive charge after contacting Teflon. However, in the case of wollastonite and feldspar there are no distinguished changes between the non-conditioned and conditioned samples since the work functions for these two minerals are less than for Teflon tribocharger. Furthermore, feldspar behaves rather differently when it is treated by salicylic acid, i.e., its avidity to be charged negatively increases considerably. This means that salicylic acid may be adsorbed on feldspar more easily than on quartz and wollastonite during wet conditioning. These results disclose a possibility for separating wollastonite from feldspar and quartz. Finally, it must be added that although benzoic and salicylic acids both contain carboxyl groups attached to aromatic rings, their influence on mineral surfaces cannot be the same. This could be because of the fact that they hold different activation energies, solubilities, melting points and densities, so their effects are different on mineral surfaces.

Generally, the adsorption of different cationic and anionic reagents on mineral surfaces increases and decreases the concentration ratio of the charge carriers. The ratio of $n_{e} / n_{p}$ changes and the Fermi energy level shifts. Therefore the avidity of mineral surfaces to accept or donate electrons changes. These kinds of adsorption influence the conductivity of minerals as well since the conductivity of semi-conducting components is a function of concentrations of charge carriers. The effect of chemicals on conductivity was already studied and described for different mineral samples [16]. Based on the physical or chemical adsorption and also on chemical components, the mineral surfaces can behave differently. Normally, when inorganic chemicals are considered the mineral surfaces change due to the formation of new surface compounds. However, this could happen when the organic chemicals are present due to chemi-sorption of the reagents.

The electronic transport in two different bands at different energy levels for semi-conductor gives a possibility to have different reactions within a solution. The exchange of the charges between a semi-conductor and ions in solution normally takes place by tunnelling [40]. 
However, there will be an easy exchange with free electrons when the energy level of an ion lies near that of the conduction band edge of a semi-conductor. On the other hand, the exchange of free holes will easily take place if this energy level lies near the energy of the valence band edge. Neither carrier exchanges readily when the ion level is deep within the forbidden gap.

\section{CONCLUSIONS}

The experimental results lead to the following conclusions:

- Dielectric constants for quartz, feldspar and wollastonite were found to be about 4.4, 5.36 and 5.65 respectively. Their estimated electrical conductivities are 58,710 and $580 \mathrm{nS} \mathrm{m}^{-1}$. These values reveal that the electro-physical properties of mineral samples are substantially similar which hinders utilisation of the conductive induction and corona charging techniques for separating these minerals from each other. On the other hand, the dielectric and conductivity values point out that wollastonite and feldspar must behave similarly.

- The triboelectric charge attributes of minerals disclose that they possess negative charge characteristics when in contact with metals. However, PVC imparts positive charges on all mineral samples because of its highest value of the work function. Therefore minerals must possess values of the work function lower than PVC. Furthermore, monitoring the charges indicates that more reliable and reproducible charges with considerably higher magnitudes can be imparted by cyclones. So the use of cyclones for triboelectric separation is suggested.

- The magnitude of the work function of mineral samples were estimated based on charges acquired by them after contacting different tribochargers. Accordingly the values of the work function found in the range from 4.39 to $4.6,4.36$ to 4.58 , and 4.35 to $4.48 \mathrm{eV}$ for quartz, feldspar and wollastonit, respectively.

- Teflon is found to be able to impart negative charge on quartz and positive one on feldspar and wollastonite when both minerals and tribochargers are heated, hence, the separation of quartz from others can be achieved. 
- The separation results after treating minerals with organic agents showed that again Teflon tribocharger could be the best for separating feldspar from wollastonite when minerals are treated by salicylic acid. In addition the triboelectric separation characteristics of quartz when Teflon was the tribocharger completely changed after treating it with sodium stearate and sodium oleate.

- Despite the fact that the results cannot be easily interpreted, they suggest a possibility to separate a mixture of quartz, wollastonite and feldspar from each others by using Teflon cyclone tribocharging device. However, two stages are recommended. First, quartz can be separated from the others without any conditioning and in the second stage the separation of feldspar from wollastonite could be achieved after conditioning the minerals with salicylic acid.

\section{References}

[1] Ralston, O. C., Electrostatic separation of mixed granular solids, Elsevier, Netherlands, 1961.

[2] Fraas, F., Electrostatic separation of granular materials, U. S. Bureau of Mines, Bulletin 603, 1962.

[3] Carta, M., Alfano, G., Carbini, P., Ciccu, R. and Del Fa, C. (1981). J. Electrostatics, 10, 177.

[4] Ciccu, R., Ghiani, M. and Ferrara, G. (1993). KONA, 11, 5.

[5] Manouchehri, H. R., Hanumantha Rao, K. and Forssberg, K. S. E. (2000). Minerals and Metallurgical Processing, 17(1), 23.

[6] Manouchehri, H. R., Hanumantha Rao, K. and Forssberg, K. S. E. (2000). Minerals and Metallurgical Processing, 17(3), 139.

[7] Lowell, J. and Rose-Innes, A. (1980). Advanced in Physics, 29(6), 947.

[8] Tromp, L., Triboelectrification, Delft Tech. University, Delft, Netherlands, 1993.

[9] Kwetkus, B. A. (1998). Particulate Science and Technology, 16(1), 55.

[10] Carta, M., Ciccu, R., Del Fa, C., Ferrara, G., Ghiani, M. and Massacci, P., Proc. 10th IMPC, IMM, London, UK, 1973, p. 349.

[11] Loeb, L. B., Static Electrification, Springer-Verlag, Berlin, 1957, p. 41.

[12] Kelly, E. G. and Spottiswood, D. J. (1989). Minerals Engineering, 2(1), 33.

[13] Kakovsky, I. A. and Revnivtzev, V. I., Proc. 5th IMPC, IMM, London, UK., 1960, p. 775.

[14] Pearse, M. J. and Pope, M. I. (1977). Powder Technology, 17, 73.

[15] Zhou, G. and Moon, K. (1994). Canadian J. Chemical Engineering, 72, 78.

[16] Manouchehri, H. R., Hanumantha Rao, K. and Forssberg, K. S. E. (1999). Minerals and Metallurgical Processing, 16(3), 14.

[17] Plaksin, I. N., Shafeyev, R. S. and Chanturia, V. I., Proc 8th IMPC, Leningrad, 1968, S-3.

[18] Carta, M., Ciccu, R., Del Fa, C., Ferrara, G., Ghiani, M. and Massacci, P. (1973). Proc. 9th IMPC, Ustav Pro Vyzkum Rud, Prague, 4, p. 47.

[19] Owada, S. and Yasukawa, K., Proc. 19th IMPC, SME, Littleton, Colorado, 1995, 2, p. 173.

[20] Fraas, F. and Ralston, O. C. (1948). Trans. Am. Inst. Mining and Met. Engrs, 12(4), 240. 
[21] Landau, L. D. and Lifshitz, E., Electrodynamic of continuous Media, Pergamon Press, New York, 1982, p. 34.

[22] Gossens, K., Van Biesen, L., Boven, A. and Castelain, P., Proc 6th International Conference on Dielectric Materials, Measurements and Applications, Short Run Press Ltd., UK., 1992, p. 73.

[23] Kumar, K. K. and Sideshmukh, L. (1996). Indian J. Pure and Appl. Physics, 34(8), 559.

[24] Fraas, F. and Raltson, O. C., Dielectric Constant in Air-Ambient Electrostatic Separation, U. S. Bureau of Mines, R.I. 4278, 1948.

[25] Church, R. H., Webb, W. E. and Salsman, J. B., Dielectric Properties of Low-Loss Mineral, U. S. Bureau of Mines, R.I. 9194, 1989.

[26] Parkhomenko, E. I., Electrical Properties of Rocks, Plenum Press, New York, 1982.

[27] Lawver, J. E. (1960). The Mines Magazine, 20.

[28] Mola, E. E. and Vicente, J. L. (1986). Surface Science, 172, 553.

[29] Ciccu, R. and Foreman, W. E. (1968). Industria Mineraria, 8, 525.

[30] Harper, W. R. (1961). Soc. Chem. Industry Monograph, 3, 115.

[31] Akande, A. R. and Lowell, J. (1987). J. Phys. D: Appl. Phys., 20, 565.

[32] Greason, W. D. and Inculet, I. I., Proc. IEEE-IAS Annual Conf., 1975, p. 428.

[33] Fomenko, V. C., Emission Properties of Materials, U.S. Department of Commerce, JPSR 56697, Springfield, Virginia, 1972.

[34] Dean, J. A., Lange's Handbook of Chemistry, McGrraw-Hill, Inc., N.Y, 1992, p. 452.

[35] Weast, R. C., CRC Handbook of Chemistry and Physics, CRC Press, Cleveland, Ohio, 1977, p. C-180.

[36] Ciccu, R. (1981). Atti. Fac. Ing. Univ. Cagliari, Univ. of Cagliari, Italy, 9(2), 139.

[37] Lee, L. H., Fundamentals of Adhesion, Plenum Press, New York, 1991.

[38] Gallo, C. F. and Lama, W. L. (1977). IEEE Trans. on Industry Appll., IA-12(1), 7.

[39] Parks, G. A., Jindal, B. K. and Anderson, J. (1966). Trans. Soc. Min Eng. AIME, 235(4), 451.

[40] Williams, R. (1976). J. Vac. Sci. Technol., 13(1), 12.

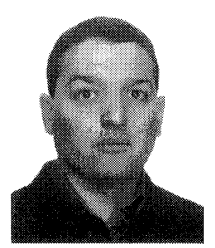

Hamid-Reza Manouchehri obtained his B.Sc. degree in Mining Engineering from the Faculty of Engineering, Tehran University, Tehran, Iran, in 1987. After graduation he joined MahabGhods Consulting Engineering and worked there in the field of Geotechnique for 1.5 years. In 1991 he received his M.Sc. degree in Mining Engineering with honours, again from the Tehran University. Subsequently he worked as an expert and later as a senior expert in mining and mineral processing for the Ministry of Mines and Metals, Iran, and also as a consultant for several private companies during 1991-1995. H. R. Manouchehri joined the Division of Mineral 
Processing, Luleå University of Technology, Luleå, Sweden in September 1995 and obtained his $\mathrm{Ph} . \mathrm{D}$. degree in mineral processing in March 2000.

Dr. Manouchehri published several papers in Farsi on processing of industrial minerals, chromite beneficiation, copper, zinc and lead mining and beneficiation, bioleaching of gold and others. He also published ten papers in English in the field electrical separation of minerals. He is the recipient of the 2000 Taggart Award from SME and is currently engaged as a consultant for Eriez Magnetics. 